\title{
Twinned dendrite growth in binary aluminum alloys
}

\author{
M.A. Salgado-Ordorica*, M. Rappaz \\ Laboratoire de Simulation des Matériaux, Ecole Polytechnique Fédérale de Lausanne, EPFL-STI-IMX-LSMX, \\ Station 12, CH-1015 Lausanne, Vaud, Switzerland
}

Received 24 June 2008; received in revised form 24 July 2008; accepted 26 July 2008

\begin{abstract}
The formation of twinned dendrites (feathery grains) in binary $\mathrm{Al}-\mathrm{Zn}, \mathrm{Al}-\mathrm{Mg}, \mathrm{Al}-\mathrm{Cu}$ and $\mathrm{Al}-\mathrm{Ni}$ alloys has been studied in specimens directionally solidified under identical thermal conditions, i.e. $G \approx 100 \mathrm{~K} \mathrm{~cm}^{-1}, v \approx 1 \mathrm{~mm} \mathrm{~s}^{-1}$, and with slight natural convection in the melt. The influence of the solute element nature and content has been found to be of less importance than previously reported since feathery grains were formed in all four alloys, regardless whether the alloying elements are hexagonal close packed ( $\mathrm{Zn}$ and $\mathrm{Mg}$ ) or face-centered cubic with a high $(\mathrm{Ni})$ or low $(\mathrm{Cu})$ stacking fault energy. A detailed analysis confirmed that twinned dendrites grow along $\langle 110\rangle$ directions in all four cases, with a complex branch morphology made of up to six to nine arms. Surprisingly, at high $\mathrm{Zn}$ or $\mathrm{Mg}$ compositions for which regular dendrites grow along $\langle 110\rangle$ instead of $\langle 100\rangle$, [Gonzales F, Rappaz M. Metall Trans A 2006; 37: 2797. [1]] no twinned dendrites could be formed. In terms of both the growth kinetics advantage of twinned dendrites over regular ones and the associated tip shape, some experimental evidence seems to contradict the doublon conjecture suggested by Henry [Henry S. PhD thesis, Ecole Polytechnique Fédéral de Lausanne, 1999. [21]], at least for the solute compositions studied in the present work.
\end{abstract}

(C) 2008 Acta Materialia Inc. Published by Elsevier Ltd. All rights reserved.

Keywords: Directional solidification; Aluminum alloys; Dendrite formation; Twin boundary

\section{Introduction}

Twinned dendrite growth in semi-continuous casting of aluminum alloys has been studied over the past 60 years $[3,4]$. Although the necessary conditions for the formation of this so-called feathery structure are fairly well known, i.e. relatively high thermal gradient $\left(G \approx 100 \mathrm{~K} \mathrm{~cm}^{-1}\right)$ and solidification speed $\left(v \approx 1 \mathrm{~mm} \mathrm{~s}^{-1}\right)$, and the presence of convection, the actual mechanisms of nucleation and growth of this peculiar dendrite morphology have still to be clarified.

As shown in Fig. 1 for a directionally solidified (DS) $\mathrm{Al}-10 \mathrm{wt} . \% \mathrm{Zn}$ alloy, feathery grains, each made of an ensemble of twinned columnar dendrites, nucleate at some point in an ingot (small circles). As regular dendritic mor-

\footnotetext{
* Corresponding author. Tel.: +41 21693 2979; fax: +41 216935890.

E-mail addresses: mario.salgado@epfl.ch (M.A. Salgado-Ordorica), michel.rappaz@epfl.ch (M. Rappaz).
}

phologies, twinned dendrites then grow and expand laterally by a branching mechanism and rapidly invade the whole volume of the ingot, overgrowing ordinary columnar grains. As there is a small but systematic misorientation between each new twinned dendrite trunk, most probably due to solute gradients, feathery grains exhibit the characteristic fan-shaped morphology seen in Fig. 1.

Unlike faceted growth of crystals, for which the role of twins is fairly well established [5], very little is known about twin nucleation during the solidification of non-faceted alloys. Atomistic-scale simulations indicate that many stacking faults can form in face-centered cubic (fcc) metals near the diffuse solid-liquid interface [6,7], but these faults, which would normally induce twins during growth, are quickly eliminated by atomic rearrangement within the solid phase. Since twinned dendrites are usually observed when convection is present, it has been suggested that their formation is enhanced by shearing forces in the melt [8]. However, this is doubtful if one makes a quick estimate of the required shear forces. On the other hand, hexagonal 


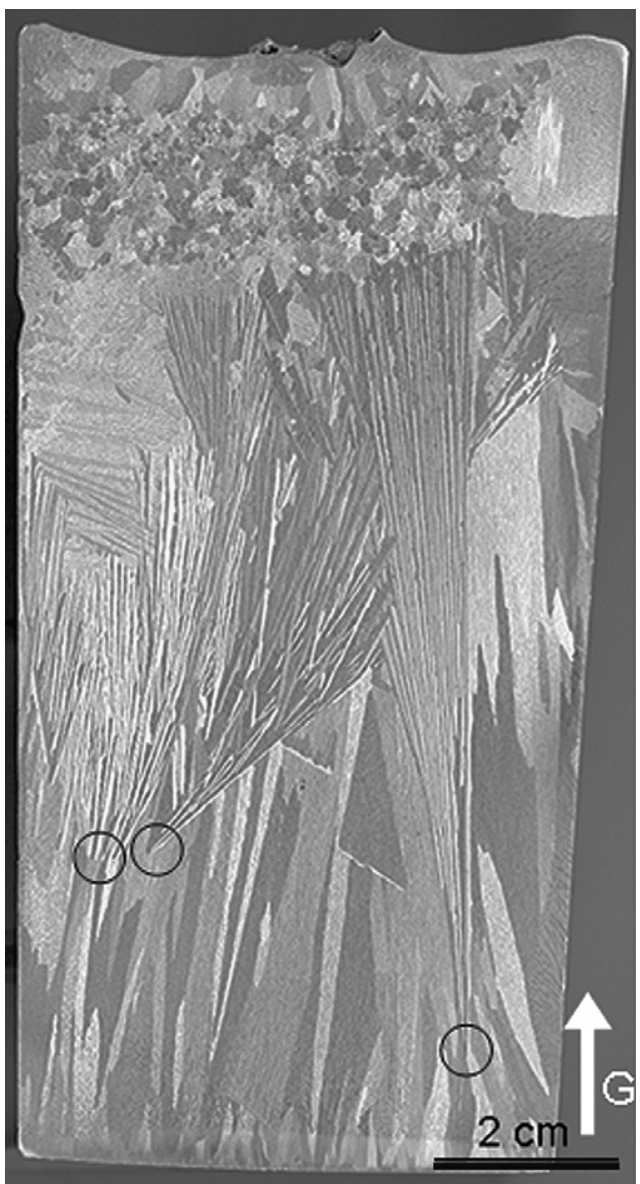

Fig. 1. Longitudinal section of a typical feathery grain structure observed in a DS Al-10 wt.\% $\mathrm{Zn}$ specimen. The location where these grains first appear in this section, which is probably close to their nucleation center, is indicated with small circles.

close packed (hcp) solute elements or fcc elements with a low stacking fault energy could possibly promote the formation of such morphologies. Unfortunately, most reported results on twinned dendrites have dealt with industrial alloys and the effect of individual alloying elements on twinning occurrence has therefore not been assessed.

From a general point of view, once it has nucleated, a feathery grain grows and forms a sequence of alternating twinned and untwinned lamellae that can appear very disoriented with respect to $G$. These lamellae are separated by an alternating sequence of straight/coherent and wavy/ incoherent (111) twin planes. Henry et al. $[9,10]$ gave a clear description of how these lamellae form: they result from the growth of twinned dendrite trunks split in their center by a (111) plane and constrained to grow along one $\langle 110\rangle$ direction contained in this plane. To explain their favorable growth kinetics over regular dendrites, several twinned dendrite tip morphologies have been proposed. Eady and Hogan [11] suggested a grooved dendrite tip that satisfies the Young-Laplace equation with the twin boundary energy $\gamma_{\mathrm{t}}$ and the solid-liquid interfacial energy $\gamma_{\mathrm{s} \ell}$. Later, however, Wood et al. [12] proposed a sharp (or edgy) dendrite tip, arguing that torques are involved when $\gamma_{\mathrm{s} \ell}$ is anisotropic. This explanation can probably be ruled out due to the weak anisotropy of $\gamma_{\mathrm{s} \ell}$ measured in aluminum alloys [13]. Recently, Henry [2] suggested the possibility of the existence of a doublon morphology, which is perfectly compatible with a weak anisotropy of $\gamma_{\mathrm{s} \ell}$ (see Fig. 2a). Although such morphologies have been observed experimentally [14] or predicted theoretically $[15,16]$ in two dimensions (2D) only, they could be induced and stabilized in 3D by the twin plane.

The twinned dendrite doublon proposed by Henry [2] (Fig. 2a) is initiated by the small groove necessary to accommodate the twin boundary energy [11]. As solute piles up in the groove, this makes it recede down to nearly the solidus temperature of the alloy. The doublon conjecture was originally supported by experimental evidence obtained in fairly dilute industrial alloys. (i) In a section parallel to the twin plane, growth seemed to be cellular instead of dendritic since no secondary arms were clearly observed (see Fig. 2b). The in-plane spacing $\lambda_{\|}$of these cells, on the order of the secondary dendrite arm spacing, was much smaller than the distance $\lambda_{\perp}$ separating two rows of twinned dendrite trunks measured in a direction perpendicular to the twin plane (Fig. 2c). The very anisotropic rejection of solute associated with the growth of $\langle 110\rangle$ doublons aligned in a (111) plane could explain that $\lambda_{\|} \ll \lambda_{\perp}$. (ii) As less solute is rejected in this case, this will also give a growth advantage of twinned doublon dendrites over regular dendrites. (iii) The strong solute gradient near the doublon and perpendicular to the twin plane could explain the gradual misorientation associated with the successive branching of new twinned trunks. (iv) A copper concentration close to the nominal composition $C_{0}$, and not to $k_{0} C_{0}$ where $k_{0}$ is the partition coefficient, was measured in an $\mathrm{Al}-4.3 \mathrm{wt} . \% \mathrm{Cu}-$ $0.3 \mathrm{wt} . \% \mathrm{Mg}$ alloy close to the twin plane by highresolution transmission electron microscopy (HRTEM) [2].

With this background in hand, the objectives of the present work are first to evaluate, in simple binary aluminum alloys, the effects of the nature and amount of solute elements. To that end, four solute elements have been selected: $\mathrm{Zn}, \mathrm{Mg}, \mathrm{Cu}$ and $\mathrm{Ni}$. $\mathrm{Zn}$ and $\mathrm{Mg}$ are two hcp elements which could favor the formation of stacking faults. Moreover, Gonzales and Rappaz have shown recently that an increasing amount of $\mathrm{Zn}$ in $\mathrm{Al}$ modifies the anisotropy of $\gamma_{\mathrm{s} \ell}$ and makes regular dendrites change their growth direction from $\langle 100\rangle$ to $\langle 110\rangle[1]$. $\mathrm{Cu}$ and $\mathrm{Ni}$ are two fcc elements with, respectively, low $\left(41 \mathrm{~mJ} \mathrm{~m}^{-2}\right)$, and high $\left(240 \mathrm{~mJ} \mathrm{~m}^{-2}\right)$ stacking fault energy [17]. The second objective is to confirm the crystallographic growth directions of twinned dendrites in these binary alloys and in particular to compare them with those of regular dendrites in the Al- $\mathrm{Zn}$ system. Finally, some experimental evidences will be presented which seem to contradict the doublon conjecture of Henry et al. $[2,10]$. 

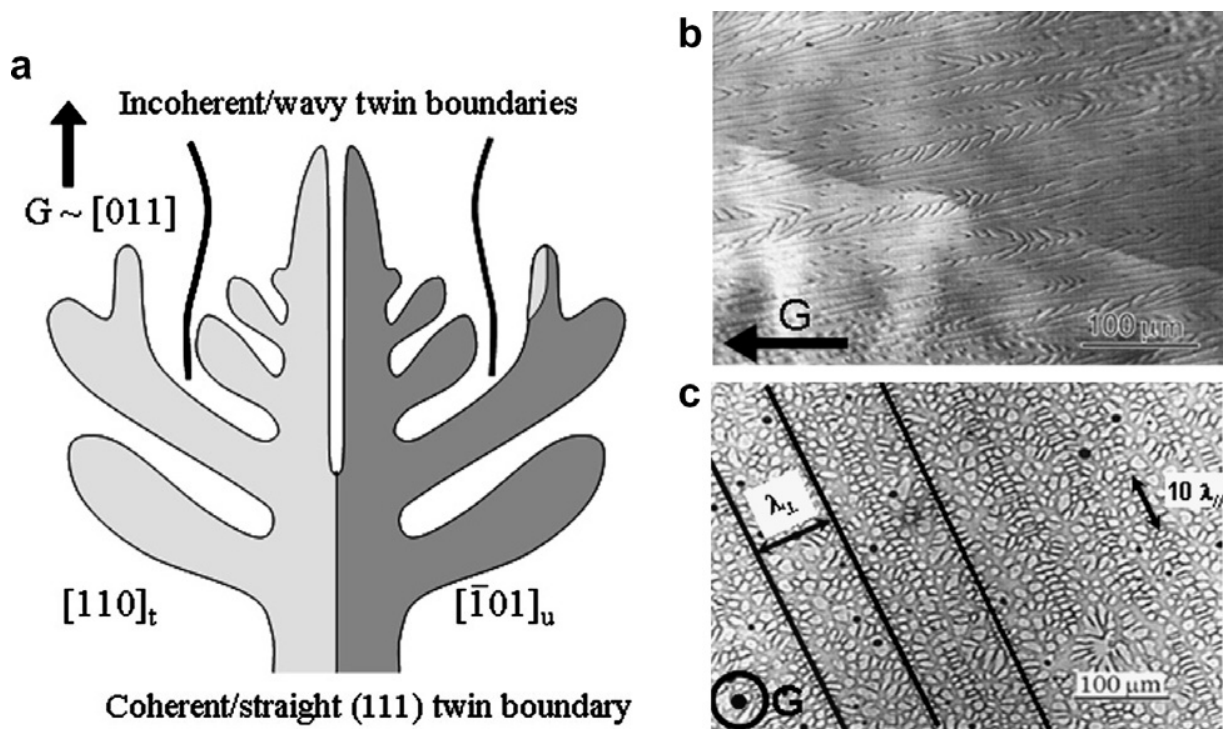

Fig. 2. (a) Twinned dendrite with a doublon morphology showing twinned and untwinned lateral arms projected in a plane perpendicular to the twin plane. A liquid channel remains in the trunk center and solidifies close to the solidus. The coherent twin plane trace appears as a line in this section

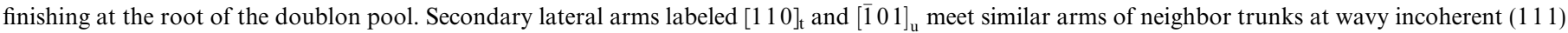
twin boundaries. (b) Section of an $\mathrm{Al}-0.11 \mathrm{wt} \% \mathrm{Cu}$ specimen nearly parallel to the twin plane (the trace of the twin plane appears as a wavy line separating dark and light grey regions). Primary trunks appear as cells with a very small primary spacing $\lambda_{\|}$and a few side branches. (c) Cross-section of the same specimen showing the drastically different primary spacings $\lambda_{\|}$and $\lambda_{\perp}$ measured parallel and perpendicular to the twin planes (identified with dark lines) $[2,10]$.

\section{Experiments and methods}

\subsection{Directional solidification}

Al-X binary alloys, where $\mathrm{X}=\mathrm{Zn}, \mathrm{Mg}, \mathrm{Cu}$ or $\mathrm{Ni}$, were prepared from pure elements ( $99.995 \mathrm{wt} \% \%$ purity) with the following compositions ranges: $10-70 \mathrm{wt} . \%$ for $\mathrm{Zn}, 5-$ 20 wt. $\%$ for $\mathrm{Mg}, 10 \mathrm{wt} . \%$ for $\mathrm{Cu}$ and $2.5 \mathrm{wt} . \%$ for $\mathrm{Ni}$ (specific values of the composition will be given in the text). Chemical composition analyses were performed by means of a Phillips XLF30 field emission gun scanning electron microscope (FEG SEM) coupled with an energy-dispersive spectrometer. For more accurate chemical analyses of regions near the twin plane, an electron microprobe microanalyzer (JEOL 8200 superprobe) was used.

The DS installation described elsewhere [1,9] was adapted to produce nearly cylindrical ingots $55 \mathrm{~mm}$ in diameter and $80 \mathrm{~mm}$ high. The stainless steel mold with a lateral surface $2 \mathrm{~mm}$ thick was closed at the bottom by a thin sheet $(0.5 \mathrm{~mm}$ thick). The interior of the mold was coated with a thin film of boron nitride in order to prevent $\mathrm{Fe}$ contamination of the melt and facilitate extraction of the specimen after solidification. The outer lateral surface of the mold was tightly surrounded by a heating wire, itself surrounded by a fairly thin layer of fiber glass wool. Three K-type thermocouples were introduced in the melt near the wall of the mold at 1,5 and $30 \mathrm{~mm}$ from the bottom plate and connected to a NetDAQ Data Acquisition System.

For each experiment, the mold was first preheated by the heating wire to $70^{\circ} \mathrm{C}$ above the liquidus temperature of the alloy. The melt was then poured into the mold and allowed to rest for $5 \mathrm{~min}$ in order to eliminate forced convection. After that, the external heating source was shut down and a water aspersion located underneath the mold bottom surface was turned on $\left(800-32001 \mathrm{~h}^{-1}\right)$. Except for the case of the $\mathrm{Al}-\mathrm{Mg}$ alloy, for which the segregation of the lighter $\mathrm{Mg}$ element can induce upward solutal convection, the three other alloy types correspond to stagnant situations in a strictly vertical thermal gradient. Under the present conditions, a slight thermal convection is expected due to the reduced insulation of the lateral mold walls.

After solidification, longitudinal and transverse sections, i.e. parallel and perpendicular to $G$, respectively, were obtained from regions located at about $35 \mathrm{~mm}$ from the bottom of the solidified ingots. Both sections were mirror polished and etched with a diluted Keller solution in order to reveal the grain structure. Once a single feathery grain was identified in both sections, the sample was polished again to mirror quality and electrochemically etched with standard solution A2-Struers (5-10 V, 5-15 s, depending on the alloy composition $[10,18]$ ) for electron back-scatter diffraction (EBSD) examination. As will be explained in more detail below, a third section parallel to the coherent twin plane was prepared for each analyzed grain. Finally, EBSD measurements and optical microscopy observations were correlated in order to identify the growth direction and the morphology of twinned dendrites.

$3 \mathrm{D}$ axisymmetric calculations of heat and mass transfer were performed using the software CALCOSOFT in order to estimate the temperature profile in the melt, in particular the axial thermal gradient $G$ and the speed of the liquidus isotherm $v$, as well as the convection induced by the small 
radial thermal gradient. These calculations were calibrated with the cooling curves measured by the three thermocouples. At about $1 \mathrm{~cm}$ from the water-cooled bottom surface, the thermal gradient $G$ near the liquidus temperature of the alloy was found to vary between 95 and $200 \mathrm{~K} \mathrm{~cm}^{-1}$, depending on the experiment, while the speed of the isotherm $v$ was in the range $1-2 \mathrm{~mm} \mathrm{~s}^{-1}$. Convection in the liquid was estimated to be of the same order of magnitude, typically $0.7 \mathrm{~mm} \mathrm{~s}^{-1}$ near the liquidus at $1 \mathrm{~mm}$ from the bottom plate.

\subsection{EBSD analysis and visualisation of growth directions}

Texture and crystallographic orientation assessment was achieved by means of a Phillips XL30 FEG SEM (nominal resolution $<2.0 \mathrm{~nm}$ at $30 \mathrm{kV}$ ) enhanced with an EBSD detector (SIT camera). The latter registered the diffraction image recorded on a phosphorus screen as a pattern of pseudo-Kikuchi lines produced by the interaction between backscattered electrons and the crystallographic planes of the sample. The pattern was automatically treated using the software Channel 5 [19] and grain structure was reconstructed by means of the Euler angle triplets $\left\{\phi_{1}, \varphi, \phi_{2}\right\}$ which provide the necessary information to transform the $(x, y, z)$ reference system of the sample into the $\langle 100\rangle$ crystallographic axes of the considered grain [20]. According to the principle of stereographic projection [21], the mean average orientation of twinned or untwinned regions was used to draw the corresponding pole figures with a program written with the software Mathematica V 6.0.

For any $\langle h k \ell\rangle$ direction, its probability of making an angle $\theta$ with the thermal gradient $G$ varies as $\sin \theta$, at least for small $\theta$ angles. Due to cubic symmetry, this probability reaches a maximum at an angle $\theta_{\max }$ and then decreases to zero at a value $\theta_{0}^{1}$ [22]. From a random population of regular grains nucleated at a surface, the grain selection mechanism operating during the growth of regular $\langle h k \ell\rangle$ dendrites is such that a $\langle k h \ell\rangle$ texture develops along the thermal gradient direction. Therefore, the dendrite growth direction can be easily identified from the texture. In the case of feathery grains, their fan-shaped morphology and their fairly low density in an ingot makes the identification of the twinned dendrite growth direction more tricky and some care is needed. A lack of precautions in previous literature dealing with twinned dendrites has lead to some confusion regarding their growth direction, in particular when simply indexing the thermal gradient direction $G$ by $\langle k h \ell\rangle$ indices in the crystallographic reference system.

For any $\langle h k \ell\rangle$ direction, its probability of making an angle $\theta$ with the thermal gradient $G$ varies as $\sin \theta$, at least for small $\theta$ angles. Due to cubic symmetry, this probability reaches a maximum at an angle $\theta_{\max }$ and then decreases to zero at a value $\theta_{0}{ }^{1}$. [22]. From a random population of regular grains nucleated at a surface, the grain selection mech-

${ }^{1}$ For $\langle 100\rangle, \theta_{\max }=\arccos (1 / \sqrt{2})$ and $\theta_{0}=\arccos (1 / \sqrt{3})$.

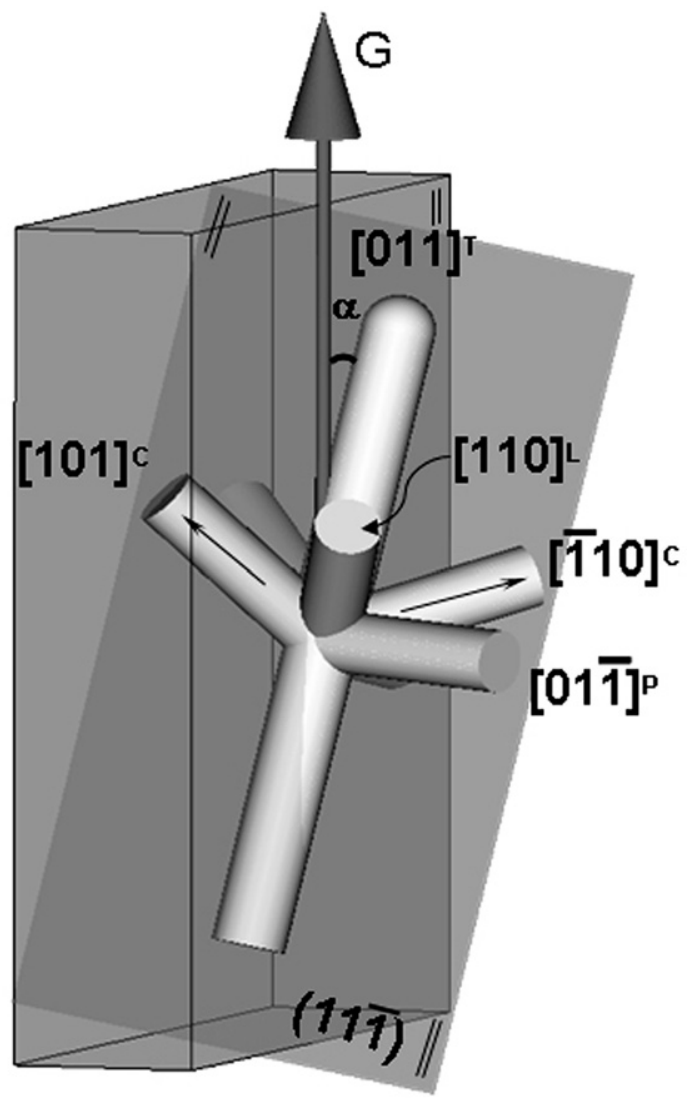

Fig. 3. 3D schematic picture of a $\langle 110\rangle$ twinned dendrite growing at an angle $\alpha$ with respect to the thermal gradient $G$.

anism operating during the growth of regular $\langle h k \ell\rangle$ dendrites is such that a $\langle k h \ell\rangle$ texture develops along the thermal gradient direction. Therefore, the dendrite growth direction can be easily identified from the texture. In the case of feathery grains, their fan-shaped morphology and their fairly low density in an ingot makes the identification of the twinned dendrite growth direction more tricky and some care is needed. A lack of precautions in previous literature dealing with twinned dendrites has lead to some confusion regarding their growth direction, in particular when simply indexing the thermal gradient direction $G$ by $\langle k h \ell\rangle$ indices in the crystallographic reference system.

Following the clear work of Henry et al. [10], we adopted the following representation in the pole figures that will be shown in the following section. The common (11) 1 ) plane of the feathery dendrites will be represented by a corresponding arc of a circle in the pole figure. The average crystallographic orientations of the twinned and untwinned parts will be shown by empty and filled squares, while common directions along the $(11 \overline{1})$ twin plane will be represented by half-filled squares. If necessary, we will identify the growth directions of the trunk as $(T)$, coplanar arms growing parallel to the plane of the twin as $\left(C_{1}\right)$ and $\left(C_{2}\right)$, the two lateral arms growing at $60^{\circ}$ from the trunk $\left(L_{\mathrm{t}}\right)$ and $\left(L_{\mathrm{u}}\right)$ and the two arms growing at $90^{\circ}$ from the trunk as $\left(P_{\mathrm{t}}\right)$ and $\left(P_{\mathrm{u}}\right)$, where the indices " $\mathrm{t}$ " and " $\mathrm{u}$ " indicate twinned and untwinned, respectively (see Fig. 3). 


\section{Results and discussion}

\subsection{Effects of solute element nature and content}

Regardless of the nature of the solute elements and under the same thermal conditions, feathery grains could be obtained in the four types of alloys investigated in the present work. The absence of a strong forced convection suggests that the combined effect of a high thermal gradient and a slight natural convection in the melt is sufficient to promote nucleation of twins and growth of twinned dendrites. Fig. 4 shows an EBSD reconstructed map of the longitudinal section of a DS Al-2.5 wt. $\% \mathrm{Ni}$ specimen. The color goes from blue to red according to the angle $\theta$ between the vertical thermal gradient $G$ and the closest $\langle 100\rangle$ direction. As can be seen, the regular columnar grains are either blue or green according to the grain selection mechanism mentioned before [22]. On the other hand, an orange-yellow grain is present at the center of the reconstructed macrostructure. This indicates that its closest $\langle 100\rangle$ direction is at about $40^{\circ}$ to $G$.

The corresponding $\langle 100\rangle$ pole figure on the right corresponds to the regular (blue-green) grains: it shows that such grains have been selected according to the growth mechanism described in Ref. [22] and are all oriented with a $\langle 100\rangle$ direction nearly parallel to the (vertical) thermal gradient. The $\langle 110\rangle$ pole figure for the yellow and orange grain clearly reveals its twinned nature: three $\langle 110\rangle$ directions belonging to the (111) twin plane (arc of a circle) are common, whereas the three other orange and yellow spots are in a symmetrical relationship with respect to this (111) plane. Note the fairly large orientation distribution, shown by the extent of each $\langle 110\rangle$ "spot" in the pole figure of this feathery grain. It is due to growth-induced misorientations in twinned and untwinned regions originated from a single nucleus, whereas the extent of the $\langle 100\rangle$ upper spot of regular (blue-green) grains is due to the orientation distribution of individual grains.

Therefore, despite the high stacking fault energy associated with $\mathrm{Ni}$ and the moderate natural convection, feathery grains have formed in this specimen. It can also be observed that this feathery grain overgrows ordinary columnar grains as it expands laterally during growth. This is, to our knowledge, the first time that twinned dendrites have been observed in the Al-Ni system.

In the case of Al-Zn alloys (e.g. Fig. 1), $\langle 110\rangle$ twinned dendrites were also observed but only up to a composition of $40 \mathrm{wt} . \% \mathrm{Zn}$. This result is rather surprising as $\mathrm{Zn}$ is an hcp solute element which should have increased the propensity to form twins. Additionally, according to Gonzales and Rappaz [1], the growth of regular $\langle 100\rangle$ dendrites at low $\mathrm{Zn}$ composition (typically $C_{\mathrm{Zn}}<25 \mathrm{wt} . \%$ ) is replaced by $\langle 110\rangle$ dendrites above $60 \mathrm{wt} . \%$. Having already $\langle 110\rangle$ regular dendrites should also have favored the growth of $\langle 110\rangle$ twinned dendrites. Such is apparently not the case. In the similar $\mathrm{Al}-\mathrm{Mg}$ system ( $\mathrm{Mg}$ is also an hop solute element), twins were observed only in an Al-7 wt.\% Mg sample, although these grains coexisted with equiaxed regular ones. In a similar industrial alloy, 5182 (mainly Al$5 \mathrm{wt} . \% \mathrm{Mg}$ ), Henry et al. [9] reported the coexistence of

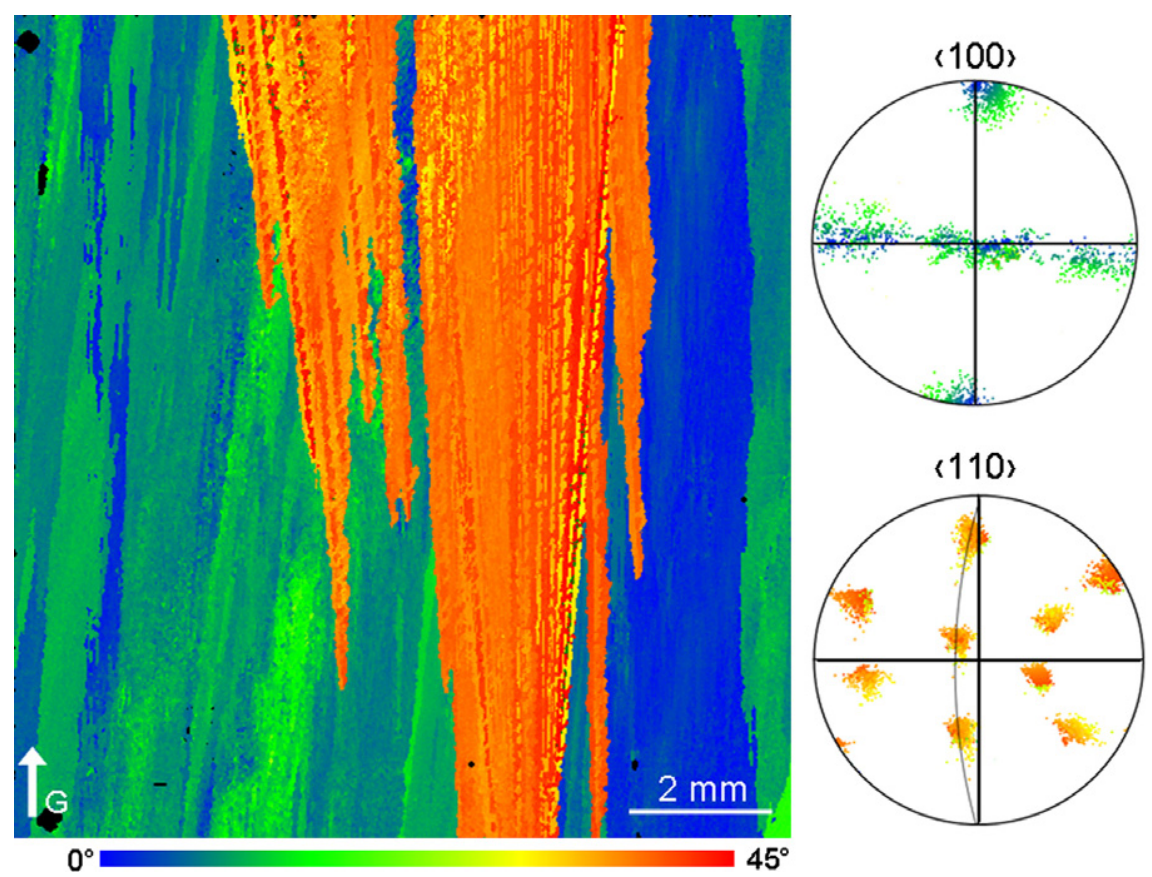

Fig. 4. EBSD false color reconstructured grain structure in a longitudinal section of a DS Al-2.5 wt.\% Ni specimen (left). The color indicates the angle between the vertical gradient $G$ and the closest $\langle 100\rangle$ direction (see scale at the bottom). On the right, the $\langle 100\rangle$ pole figure of the regular blue-green columnar grain is shown as well as the $\langle 110\rangle$ pole figure of the yellow and orange lamellae of the central grain. The stereographic projections have the same axes as the grain structure. 

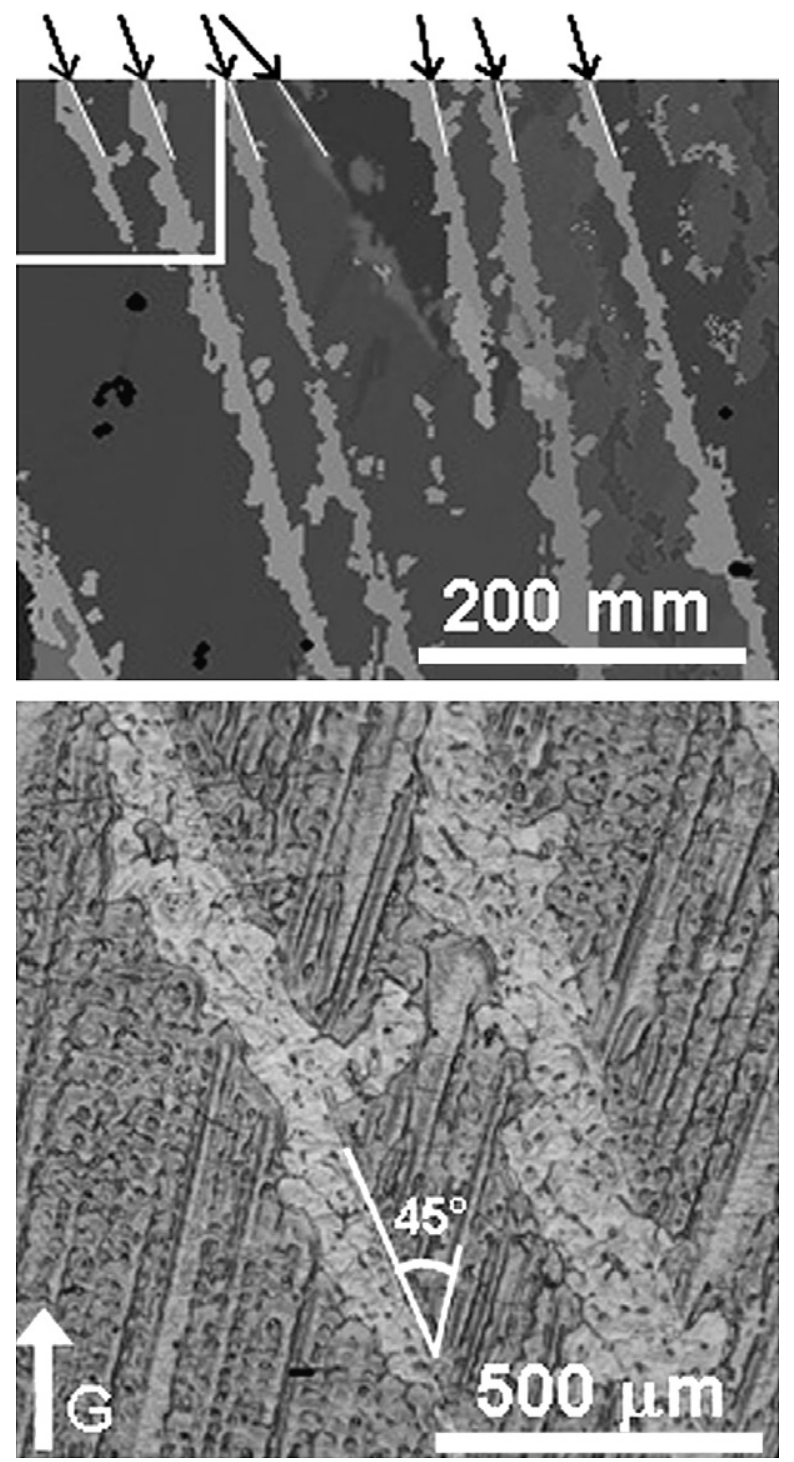

Fig. 5. EBSD reconstructed grain structure map (top) and optical micrograph of the enlarged region located in the rectangle (bottom) showing discontinuities of the coherent twin planes in the longitudinal section of a $\mathrm{DS} \mathrm{Al}-10 \mathrm{wt} . \% \mathrm{Cu}$ specimen.

columnar $\langle 110\rangle$ regular and twinned grains, but no equiaxed grains. ${ }^{2}$ From these observations, it can be concluded that: (i) the dendrite orifentation transition (DOT) from $\langle 100\rangle$ to $\langle 110\rangle$ during the growth of regular dendritic grains [1] occurs at much lower compositions in $\mathrm{Al}-\mathrm{Mg}$ compared to Al-Zn; (ii) the formation of $\langle 110\rangle$ twinned dendrites in the presence of a slight convection is not necessarily favored in alloy systems where $\langle 110\rangle$ regular dendrites normally grow without convection. It seems more influenced by the hcp solute element content, a too high composition of the alloy decreasing, however, its ability to form twins.

\footnotetext{
${ }^{2}$ Henry et al. used a higher thermal gradient and more dilute alloys, which explains why they did not observe, unlike us, a columnar-toequiaxed transition [23].
}

Although this was not the case in Al-Zn alloys, the continuity of the coherent twin planes appeared to be systematically interrupted in the other alloy systems. This phenomenon is illustrated in Fig. 5 which shows at the top an EBSD reconstructed grain structure in a longitudinal section of an $\mathrm{Al}-10 \mathrm{wt} . \% \mathrm{Cu}$ alloy. The twinned and untwinned lamellae in this figure appear as light and dark grey. Note from the traces of the (111) twin planes that at least two feathery grains are present. The alternating sequence of wavy/incoherent and straight/coherent twin boundaries can be observed in this specimen, but the latter ones identified by small arrows at the top are interrupted at several places. As the thermal gradient is vertical, it appears that the light grey part of the lamellae located "below" the coherent twin planes (i.e. at lower temperatures) frequently extend in the dark grey regions, thus making the coherent twin boundaries discontinuous. Such a region is highlighted with a white rectangle and is enlarged in the optical micrograph shown at the bottom of Fig. 5. The incursions of the light grey dendritic pattern into the upper dark grey region through the coherent twin plane can be clearly seen. As will be further explained in the next section, these observations rule out one of the arguments put forward for the formation of doublons [2]. On the other hand, as the twin plane (marked with a white line) is fairly inclined with respect to the vertical thermal gradient, the dark zone is favored for the growth of secondary arms. These arms (marked also with a white line) are nearly parallel to the longitudinal section and grow at $45^{\circ}$ from the twin plane: they do correspond to regular $\langle 100\rangle$ side arms. This shows that: (i) $\langle 110\rangle$ twinned dendrites can form regardless of the growth directions of regular dendrites, i.e. with $\langle 100\rangle$ side arms, thus confirming the results deduced on $\mathrm{Al}-\mathrm{Zn}$ and $\mathrm{Al}-\mathrm{Mg}$; (ii) twinned dendrite mor-

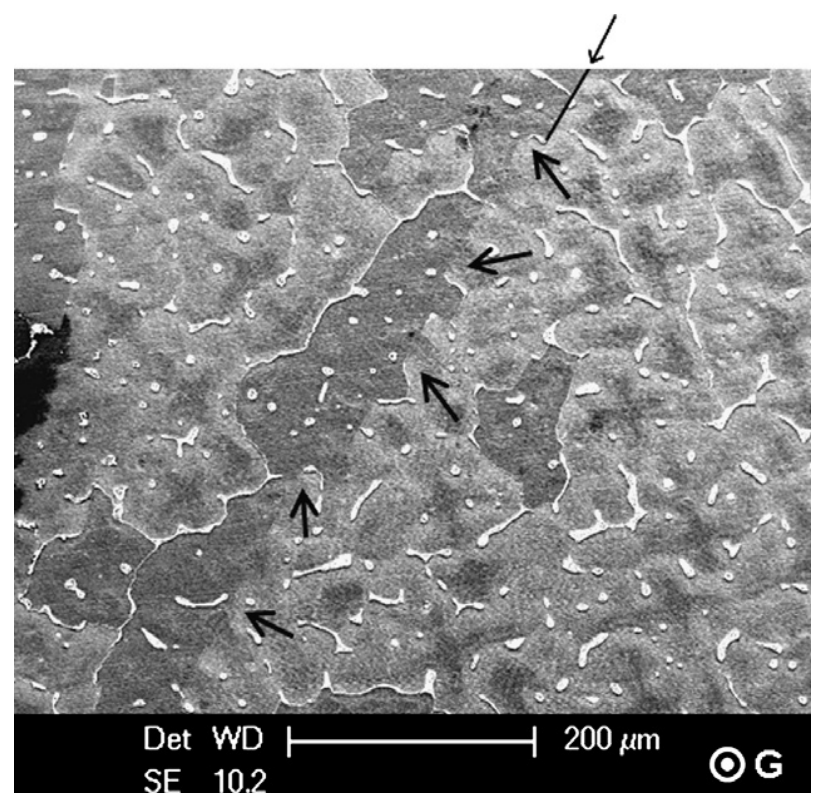

Fig. 6. Twin plane interrupted by the presence of secondary phases during solidification in a transverse section of an $\mathrm{Al}-10 \mathrm{wt} . \% \mathrm{Cu}$ alloy. 


\section{(110) pole figures}
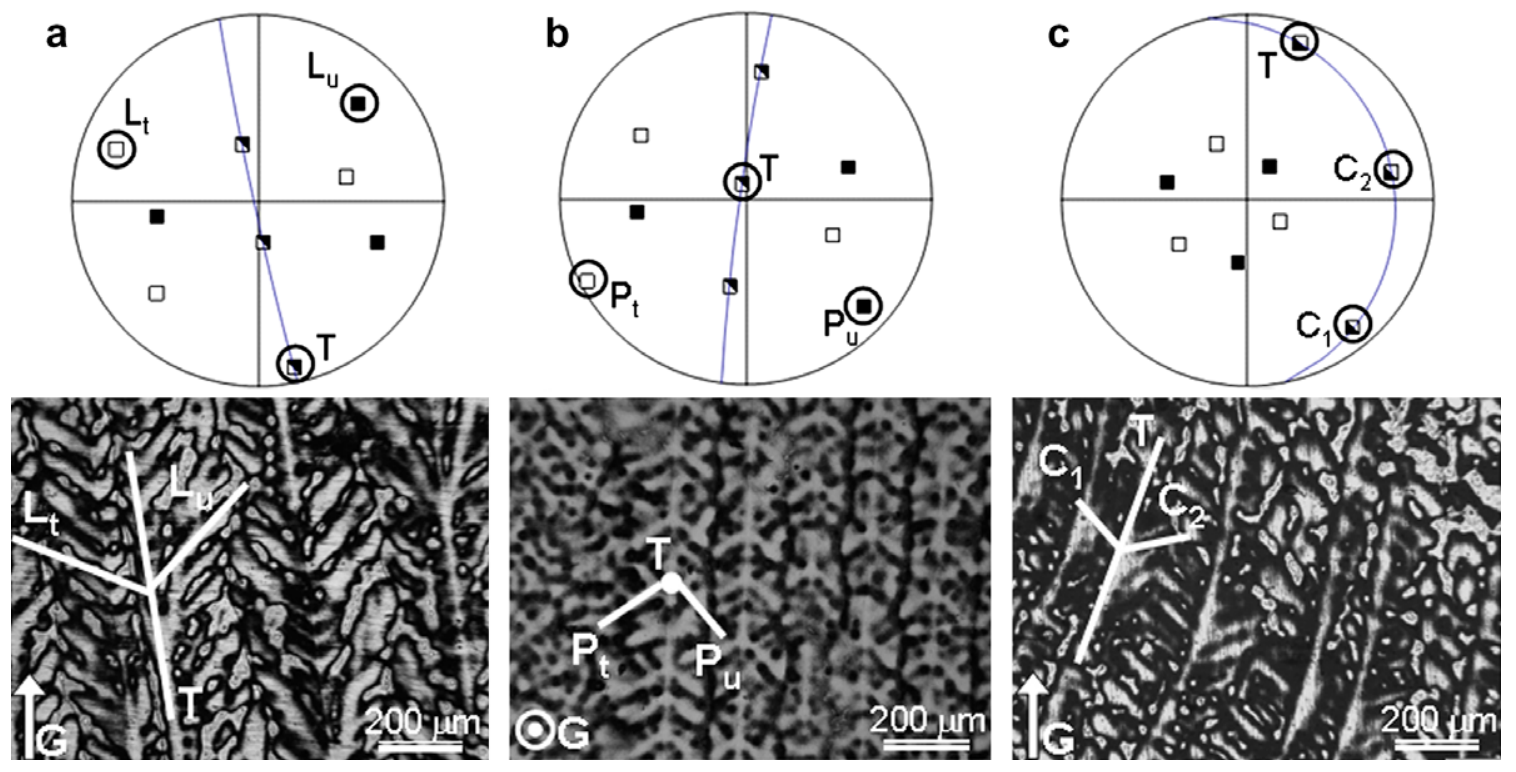

Fig. 7. Microstructures and corresponding $\langle 110\rangle$ pole figures of sections (a) parallel to $G$, (b) perpendicular to $G$ and (c) nearly parallel to the twin plane, for a DS Al-20 wt.\% Zn specimen. The direction of the twinned dendrite trunk $(T)$ and that of the main side arms $(L, P$ or $C$, see Fig. 3$)$ have been indicated in both micrographs and pole figures.

phology can be more complex than previously described and schematized in Fig. 3.

The discontinuity of the coherent twin boundary is further examined in Fig. 6 for an $\mathrm{Al}-10$ wt.\% $\mathrm{Cu}$ alloy using SEM with secondary electrons, i.e. contrast essentially topographic. The surface of the specimen which corresponds to a transverse section has been previously etched. In addition to a pore (dark region) seen on the left boundary of the micrograph, the eutectic phase appears white and the twinned/untwinned regions in dark and light grey. The position of an interrupted coherent twin is marked by a small arrow at the top. The straight parts of the twin are coherent and correspond to dendrite trunks split in two parts. At some locations identified with small arrows, one side of the twinned grain extends slightly into the other: in this case, it forms some eutectic at the twin boundary and this latter is incoherent. The eutectic forms at such regions as at the wavy incoherent boundaries outlining the regions where secondary arms meet (see Fig. 2). In this specimen, it looks as if there is some competition between the twinned and untwinned parts of the twinned dendrite trunks. This was usually not observed in the $\mathrm{Al}-\mathrm{Zn}$ alloy system with $C_{\mathrm{Zn}}<40 \mathrm{wt} . \%$, for which there is no eutectic formation. A most probable explanation for these "incursions" across the coherent twin plane will be given in Section 3.3.

\subsection{Growth directions}

Some indications about the actual crystallographic growth direction of twinned dendrites can already be inferred from the previous section, in particular in Fig. 4.
However, in this figure there is no $\langle 110\rangle$ direction exactly aligned with the vertical thermal gradient. At the same time, the growth direction of twinned dendrites is not necessarily parallel to $G$. In order to avoid any misleading interpretation, a detailed analysis based on the observations of several feathery grains in three different sections has been performed for all alloy types. Fig. 7 shows three micrographs and their corresponding $\langle 110\rangle$ pole figures for a DS Al-20 wt.\% $\mathrm{Zn}$ specimen. Since this particular feathery grain has dendrites fairly well aligned with the thermal gradient, the section parallel to $G$ (Fig. 7a) clearly shows trunks coinciding with a $\langle 110\rangle$ direction nearly in this plane (labeled $(T)$ in both the micrograph and the pole figure). However, the trunks are not exactly continuous in the section as the $\langle 110\rangle$ direction is slightly off this plane. Although the lateral arms $\left(L_{\mathrm{t}}\right)$ and $\left(L_{\mathrm{u}}\right)$ are not lying in the plane of the section, their projections are nearly at $60^{\circ}$ from the trunks.

In the case of the section perpendicular to $G$ (Fig. 7b), primary dendrite trunks appear as rows nearly parallel to the (111) plane shown as an arc in the corresponding pole figure. The side arms have complex morphologies as they can be either perpendicular side arms $\left(P_{\mathrm{t}}\right)$ and $\left(P_{\mathrm{u}}\right)$ growing parallel to the plane of the section, or $\left(L_{\mathrm{t}}\right)$ and $\left(L_{\mathrm{u}}\right)$ arms growing slightly inclined towards the observer. Tertiary arms with the $(L)$ orientation developing from $(P)$ or $(L)$ secondary branches in each twinned and untwinned region can also be seen. This makes the dendrite morphology of twinned dendrites fairly complex as three $\langle 110\rangle$ variants can develop on each side of the twin, in addition to the three common $\langle 110\rangle$ directions growing parallel to the 


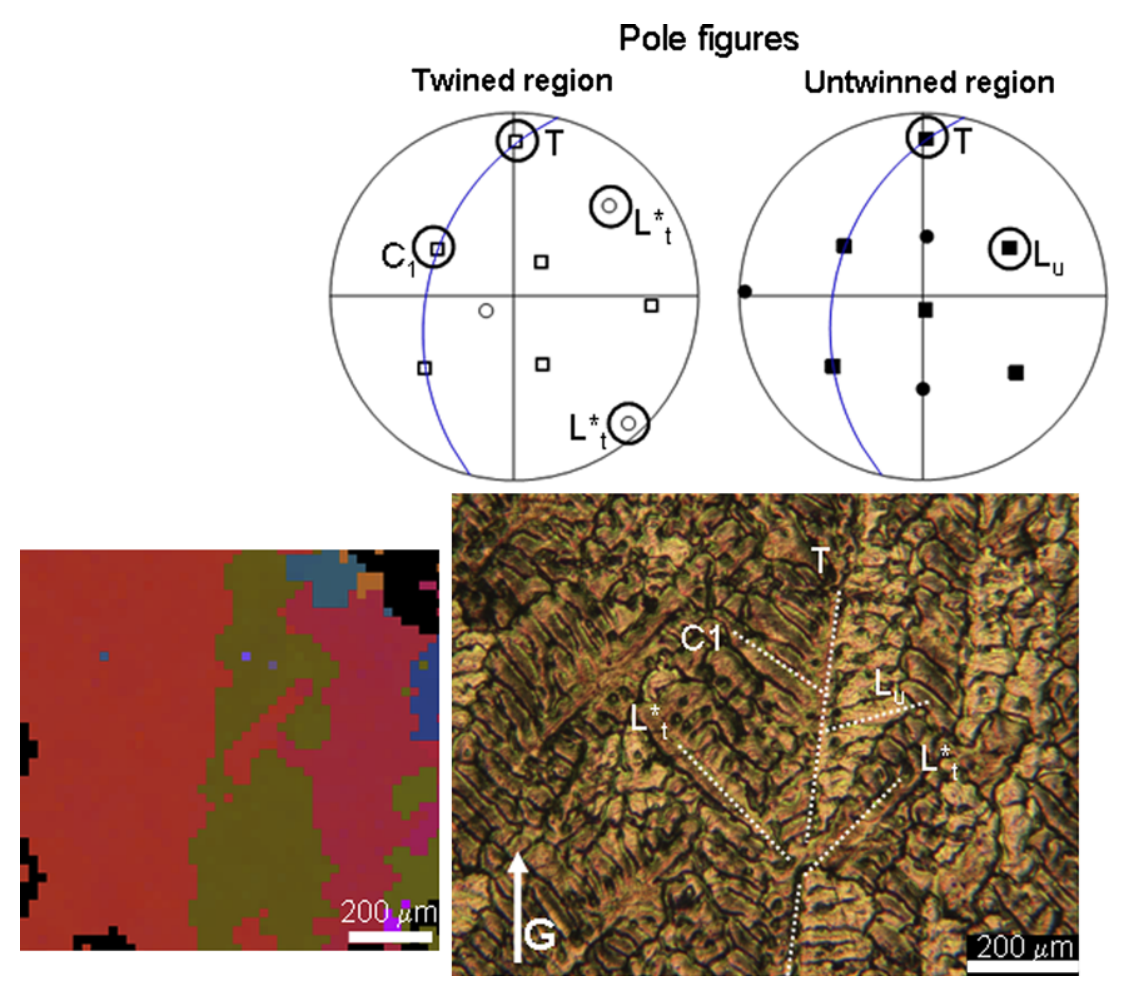

Fig. 8. $\langle 100\rangle$ side arms growing in an $\mathrm{Al}-10 \mathrm{wt} . \% \mathrm{Cu}$ alloy. The $\langle 100\rangle$ and $\langle 110\rangle$ directions are identified with circles and squares, respectively, in the corresponding stereographic projections at the top. The twinned and untwinned orientations of the grain are drawn with open and filled symbols, respectively. The EBSD false color rescontructed grain structure on the left shows the twin and untwinned parts in red and brown.

twin. On the left of the micrograph shown in Fig.7b, a different dendritic structure can be observed that has been identified to grow along $\langle 100\rangle$.

The last section, nearly parallel to the twin plane (Fig. 7c), clearly shows the two coplanar side arms $\left(C_{1}\right)$ and $\left(C_{2}\right)$ which have grown at $60^{\circ}$ from the trunk. As this section is at about $10^{\circ}$ from the twin plane (see the corresponding $\langle 110\rangle$ pole figure at the top), a slight contrast between the lower-left and upper-right parts of the micrographs can be seen: the transition line between these two regions corresponds to the trace of the (111) twin plane in this section. Unlike previous observations (see Section 1, Fig. 2b) [2,10], the coplanar arms are very well developed in this alloy on both sides of the twin plane. This makes the trunk spacing in this section $\lambda_{\|}$nearly equal to the trunk spacing $\lambda_{\perp}$ measured in Fig. 7a or b. The apparent curvature of the trunks in Fig. 7c is due to the twin plane misalignment with respect to the section.

From the three micrographs of Fig. 7, the complete 3D morphology of the twinned dendrite can be summarized (and simplified) according to the schematic view shown in Fig. 3, at least for the $\mathrm{Al}-20 \mathrm{wt} . \% \mathrm{Zn}$ and $\mathrm{Al}-7 \mathrm{wt} . \%$ $\mathrm{Mg}$ alloys. In the case of $\mathrm{Al}-\mathrm{Cu}, \mathrm{Al}-\mathrm{Ni}$ and higher solute content $\mathrm{Al}-\mathrm{Zn}$ alloys, secondary arms can also grow along $\langle 100\rangle$ as already pointed out in Fig. 5. The growth of $\langle 100\rangle$ and $\langle 110\rangle$ side arms is illustrated in Fig. 8 for a DS Al-10 wt. $\% \mathrm{Cu}$ alloy (longitudinal section). At the top to the optical micrograph, $\langle 100\rangle$ (circles) and $\langle 110\rangle$ (squares) directions for the twinned/untwinned parts are shown by open/filled symbols, respectively. The corresponding EBSD reconstructed false color map of the same zone is shown on the left (red and brown correspond to the twinned/untwinned parts, respectively). Again, the coherent twin plane is not continuous as seen from the fairly corrugated boundaries in the EBSD map. A $\langle 100\rangle$ arm (in red, twinned) was even able to grow slightly from below into the untwinned (brown) region: it is labeled $\left(L_{t}^{*}\right)$ because it is $\langle 100\rangle$ and is circled in the corresponding pole figure. In the red region, other $\langle 100\rangle$ lateral arms have also grown together with $\left(C_{1}\right)$ (also circled in the twinned part pole figure). In the untwinned region, $\left(L_{\mathrm{u}}\right)$ arms have been identified. All these features make this twinned dendrite morphology much more complex than the small sketch shown in Fig. 3, which is why no transverse section is shown for this specimen. Although the side arms are not always $\langle 110\rangle$ and the coherent twin planes can be interrupted by the growth of one side into the other region, it should be emphasized that the trunk growth direction $(T)$ identified in sections nearly parallel to the twin plane was always $\langle 110\rangle$.

\subsection{Validity of the doublon conjecture}

Among the various hypotheses suggested in the past for the growth advantage and morphology of twinned dendrites $[2,11,12]$, the formation of a groove at the tip, leading possibly to the formation of a doublon, is the most appealing. One of the arguments for the presence of doub- 
lons in feathery grains is the very anisotropic primary trunk spacing measured along the twin plane and perpendicular to it, i.e. $\lambda_{\|} \ll \lambda_{\perp}$. However, the measurements were made in fairly dilute industrial alloys. In order to further validate or invalidate the doublon conjecture, measurements of $\lambda_{\|}$ and $\lambda_{\perp}$ were performed for several feathery grains of our binary alloy systems. Fig. 7 already indicates that $\lambda_{\|}$is comparable to $\lambda_{\perp}$ in $\mathrm{Al}-20 \mathrm{wt} . \% \mathrm{Zn}$. Fig. 9 shows a transverse section of an $\mathrm{Al}-30 \mathrm{wt} . \% \mathrm{Zn}$ alloy in which the straight coherent twin planes are clearly visible. Several side arm variants are again present in this specimen, some of them corresponding to $\langle 110\rangle-(P)$ arms and others to $\langle 100\rangle$ arms. These arms appear as "V"s in this transverse cross-section, as already pointed out by Henry et al. [8]. The rows of dendrite trunks belonging to a given coherent twin plane are clearly identified, thus giving the spacing $\lambda_{\perp}$. Within a coherent twin plane it is not evident to measure $\lambda_{\|}$ but the trunks are definitely not juxtaposed to each other. Taking $\lambda_{\|}$as the spacing between two clearly identified "V"s, it is concluded that $\lambda_{\perp}$ is comparable to $\lambda_{\|}$. This experimental evidence, together with the non-continuity of the coherent twin planes seen in other specimens, are already two factors that do not support the doublon conjecture.

Another argument put forward by Henry for the formation of doublons was the solute composition measured near a coherent twin plane (see Section 1) [2]. These doublons grow with a thin liquid channel, a few microns wide, in their center. This liquid channel is enriched in solute as the temperature is lowered, and eventually solidifies with a final liquid composition close to $C_{0} / k_{0}$, i.e. a solid composition close to $C_{0}$. Since the two tips of the doublon, on both sides of the liquid channel, have a solid composition close to $k_{0} C_{0}$, a strong negative solute gradient should be measured on both sides of the coherent twin plane, after complete solidification. Although solute diffusion during and after solidification is expected to smear out such solute

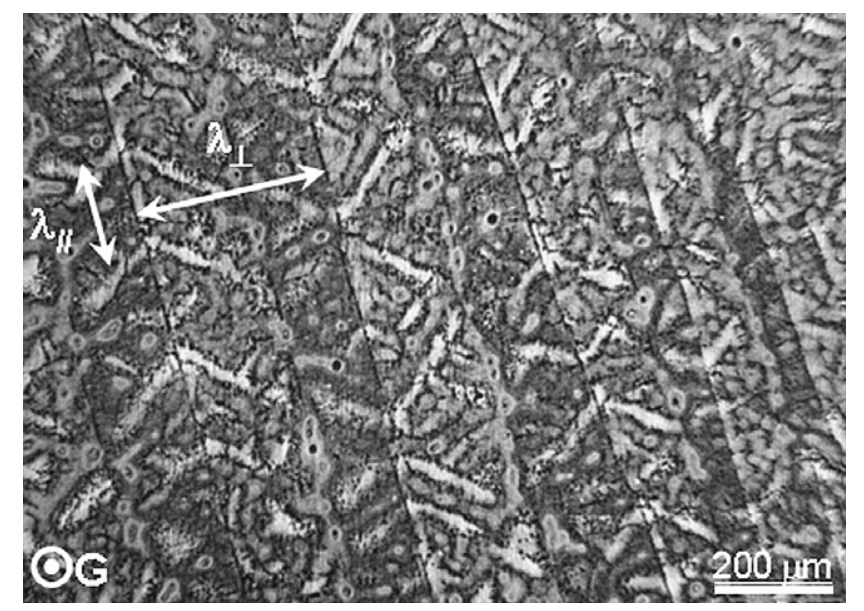

Fig. 9. Primary dendrite trunk spacing $\lambda_{\|}$and $\lambda_{\perp}$ measured in a direction parallel, respectively perpendicular, to the twin planes in a cross-section of a DS Al-30 wt.\% Zn specimen. gradients, they would definitely be opposite to the increasing solute gradients measured from the center of a regular dendrite.

In order to quantify the solute content profile across a coherent twin plane, energy-dispersive X-ray analysis (EDX) chemical analyses were performed on a cross-section of an $\mathrm{Al}-30 \mathrm{wt} . \% \mathrm{Zn}$ specimen (Fig. 10). The optical micrograph in Fig. 10a shows the alignment of trunks along the (111) twin planes with side arms that make $\lambda_{\|}$ comparable to $\lambda_{\perp}$. In a region close to the center of a twinned trunk (white dashed line), 25 EDX measurements were made over a length of $50 \mu \mathrm{m}$ along the thick black line shown in the back-scattered SEM micrograph of Fig. 10b. The solute profile measured along this line is shown in Fig. 10c. From both the absence of contrast near the twin plane evidenced in the SEM micrograph and the shape of the solute composition profile, it is deduced that no strong segregation occurs near the twin plane. Furthermore, the $\mathrm{Zn}$ composition has the standard shape associated with regular dendrites, i.e. it is slightly higher than $c_{\mathrm{s}}$, calculated

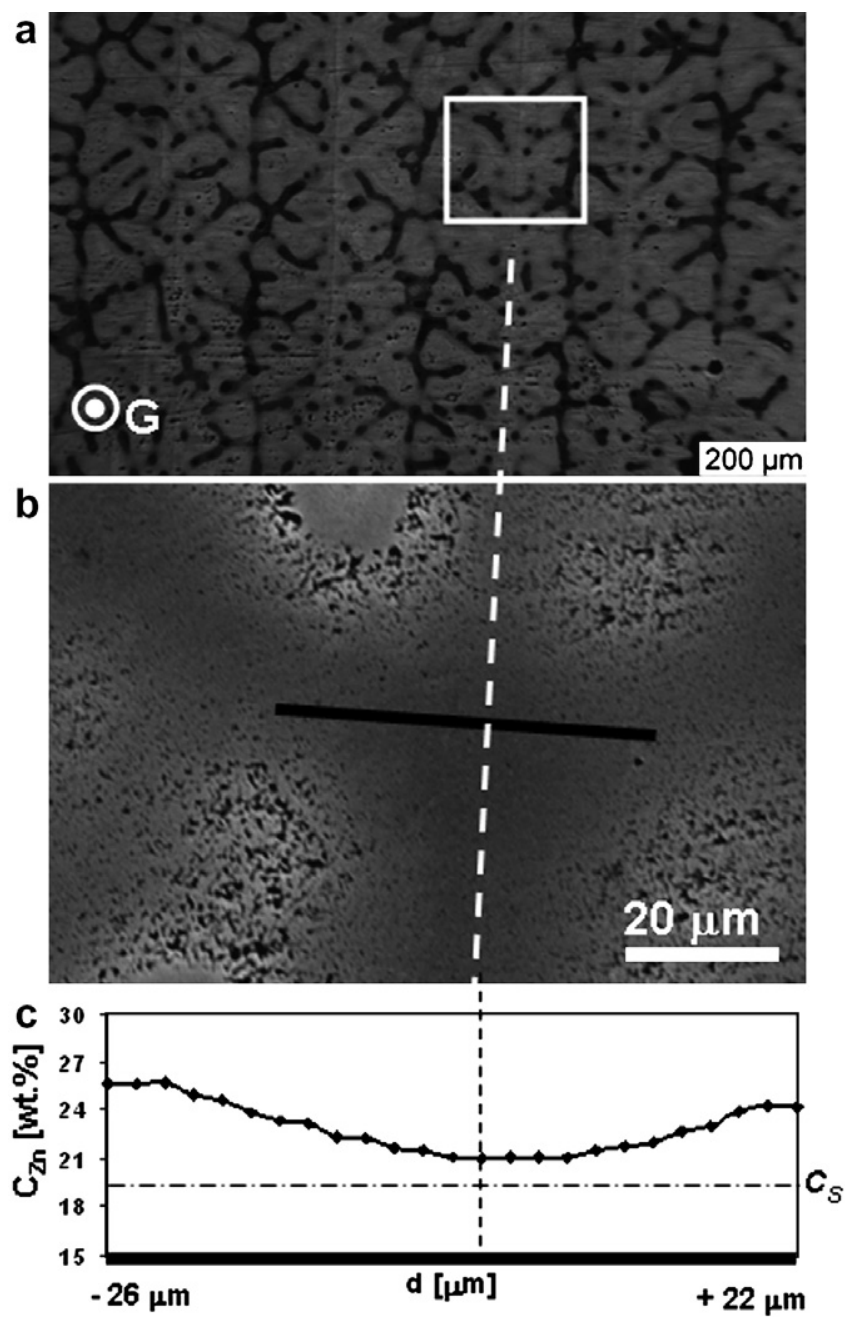

Fig. 10. (a) Optical micrograph of a DS Al-30 wt.\% $\mathrm{Zn}$ alloy in a crosssection (Keller's etching). (b) Back-scattered SEM micrograph showing the trace of the twin plane (dashed white line) and the thick black line along which the composition profile (c) was measured by EDX. 

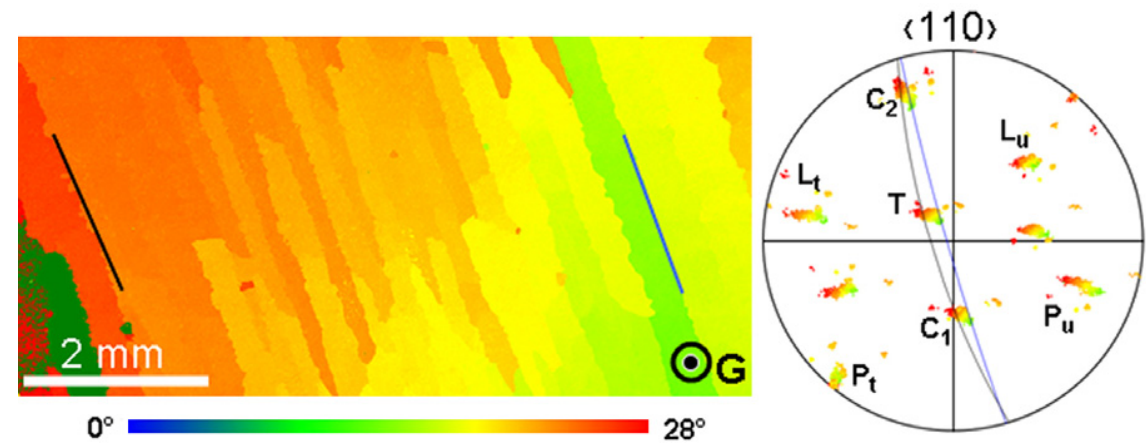

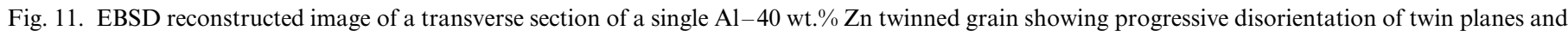

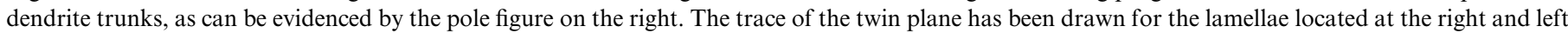
sides of the reconstructed map

with the KGT model of marginal stability [24] (dashed horizontal line in Fig. 10c), near the center of the trunk and increases as one moves away.

This finding, combined with previous ones, leads us to conclude that the doublon conjecture is not valid for high solute content $\mathrm{Al}$ alloys. However, the arguments of Henry et al. $[2,10]$ cannot be completely ruled out since they were based on low solute content alloys. Furthermore, the apparent absence of doublons in our alloys does not completely eliminate the possibility of having a grooved dendrite tip [11]. This groove could degenerate into a doublon by solute accumulation, depending on the alloy composition.

The last argument put forward by Henry et al. to support the doublon conjecture is the systematic misorientation between lamellae $[10,2]$. These misorientations, which explain the overall fan-shaped morphology of feathery grains (see Fig. 1), would be due to systematic and strong solute gradients perpendicular to the twin plane. Fig. 11 shows that such misorientations are also observed in feathery grains of the highest $\mathrm{Zn}$ content specimen, $\mathrm{Al}-40 \mathrm{wt} . \% \mathrm{Zn}$. In this transverse section, the color scale of the EBSD reconstructed map corresponds to the angle between the thermal gradient $G$, perpendicular to the figure, and the nearest $\langle 110\rangle$ direction (labeled $(T)$ in the corresponding pole figure). Although the (111) twin plane traces remain nearly parallel in this section, they show an increasing misorientation as one moves from the top-right to the bottom-left of the picture, i.e. from yellow (about $15^{\circ}$ misorientation between $G$ and $(T)$ ) to red (about $28^{\circ}$ ). Nevertheless, this is a single grain as observed in the corresponding $\langle 110\rangle$ pole figure. The axis of rotation is not exactly parallel to the normal to $G$ contained in the (111) plane. Probably because the $\langle 110\rangle$ twinned dendrite trunks are not aligned with $G$, there is also a small rotation of the twin planes along an axis parallel to $G$ as shown by the two traces of the twin planes drawn in the pole figure (blue and black for the right, respectively left, lamellae in the micrograph). Furthermore, a misorientation discontinuity of a few degrees is observed in each lamella, i.e. a sudden change of color as one moves parallel to the twin traces.
Although these misorientations are not fully understood and do not necessarily prove the existence of doublons, they are most probably linked to systematic solute gradients occurring in a specific alignment of twinned dendrite trunks, in a way similar to misorientations observed in regular dendrite arrangements [25]. Due to the specific arrangement of twinned dendrite trunks in rows, these solute gradients are essentially perpendicular to the twin planes.

\section{Conclusion}

Different binary $\mathrm{Al}$ alloys of various compositions have been investigated with solute elements having a low/high stacking fault energy ( $\mathrm{Cu}$ and $\mathrm{Ni}$, respectively) or solidifying with a hcp structure ( $\mathrm{Mg}$ and $\mathrm{Zn})$. In all alloys of the four systems, twinned dendrites can nucleate and grow regardless of the nature of the solute element, but not of the composition and as long as critical thermal and convection conditions are met. Surprisingly, twinned dendrites could not be formed in $\mathrm{Al}-\mathrm{Zn}$ at high compositions for which regular $\langle 110\rangle$ dendrites formed in the absence of convection [1]. In some specimens, it was shown that $\langle 110\rangle$ twinned dendrites can grow with $\langle 110\rangle$ and $\langle 100\rangle$ side arms. Several experimental observations do not support the conjecture of twinned dendrite doublons: discontinuity of the coherent twin planes; the existence of coplanar side arms growing parallel to the twin plane and making the in-plane trunk spacing $\lambda_{\|}$close to the perpendicular one $\lambda_{\perp}$; the absence of a strong segregation near the dendrite trunk center; and the solute profile typical of regular dendrites, i.e. solute increase from the dendrite trunk center to the periphery (for $k_{0}<1$ ). However, it should be kept in mind that the observations of Henry et al. $[2,8-10]$ were done for fairly diluted industrial alloys while the present ones have been carried out on much more concentrated binary alloys. As suggested by Eady and Hogan [11], the twinned dendrite tip probably has a groove in order to satisfy the mechanical equilibrium condition and this groove could deepen up to a doublon depending on the solute content. Further experimental work on dilute binary alloys as 
well as phase field simulations are in progress in order to verify this interpretation. In any case, a grooved or doublon-type morphology gives a clear growth advantage to twinned dendrites over regular ones. For both tip morphologies, the alignment of primary trunks along (111) planes and the associated solute gradients might be responsible of the systematic lamellae misorientation. Independently of the tip morphology of twinned dendrites, their highly branched morphology can help them propagate faster in a specimen. If there is some convection, there is a greater chance that some of them grow against the flow, i.e. grow faster.

\section{Acknowledgements}

The authors thank the staff of the Interdisciplinary Center for Electron Microscopy of the Ecole Polytechnique Fédérale de Lausanne (EPFL), and in particular Dr. Emmanuelle Boehm, for the help in the EBSD measurements. The collaboration of Jonas Vannod and Jean-Daniel Wagnière is also acknowledged.

\section{References}

[1] Gonzales F, Rappaz M. Metall Trans A 2006;37:2797.

[2] Henry S. PhD thesis, Ecole Polytechnique Fédéral de Lausanne, 1999.
[3] Herenguel J. Rev Metall 1948;45(5):339.

[4] Fredriksson H, Hillert M. J Mater Sci 1971;6:1350.

[5] Hamilton DR, Seidenticker RG. J Appl Phys 1960;31(1):1165.

[6] Burke E, Brougthon JQ, Gilmer GH. J Chem Phys 1988;89:1030.

[7] Hoyt JJ, Asta M. Phys Rev B 2002;65:214106.

[8] Henry S, Gruen G, Rappaz M. Metall Mater Trans A 2004;35:2495.

[9] Henry S, Minghetti T, Rappaz M. Acta Mater 1998;46(18):2495.

[10] Henry S, Jarry P, Jouneau P-H, Rappaz M. Metall Mater Trans A 1997;28:207.

[11] Eady JA, Hogan LM. J Cryst Growth 1974;28:129.

[12] Wood HJ, Hunt JD, Evans PV. Acta Mater 1997;45(2):569.

[13] Napolitano RE, Liu S. Phys Rev B 2004;70:214103.

[14] Akamatsu S, Faivre G, Ihle T. Phys Rev E 1995;51(5):4751.

[15] Ihle T, Müller-Krumbhaar H. Phys Rev E 1994;49(4):2972.

[16] Amar MB, Brener E. Phys Rev Lett 1995;75:561.

[17] Kelly A, Groves G. Cristallography and crystal defect. New York: Addison-Wesley; 1970. p. 258.

[18] Katrakova D. Prakt Metallogr 2001;38:10.

[19] Dingley D. J Microsc 2003;21(3):214.

[20] Randle V. Electron backscatter diffraction in materials science. Dordrecht: Kluwer Academic Publishers; 2000.

[21] Johari O, Thomas G. The stereographic projection and its applications. New York: Interscience; 1969. pp. 15-45.

[22] Gandin Ch-A, Rappaz M, West D, Adams BL. Metall Mater Trans A 1995;26:1543.

[23] Rappaz M, Gandin Ch-A. Acta Metall Mater A 1993;41(2):345.

[24] Kurz W, Giovanola B, Trivedi R. Acta Metall 1986;34:823.

[25] Rappaz M, Blank E. J Mater Sci 1987;22:896. 